\title{
A Novel Low-Cost Noncontact Resistive Potentiometric Sensor for the Measurement of Low Speeds
}

\author{
Xiujun Li and Gerard C. M. Meijer, Member, IEEE
}

\begin{abstract}
A novel low-cost sensor system for the measurement of low angular or linear speed is presented. The sensing element consists of a noncontact resistive potentiometer. The applied sliding electrode does not make mechanical contact with the resistive layer. The processing circuit consists of a very linear oscillator which converts the position quantity to a period-modulated signal. This signal can be directly read out by a microcontroller. A novel algorithm is presented which can eliminate or strongly reduce the influence of the many nonidealities, and which results in a short measurement time. The resolution of the low-cost angular speed sensor system is about $8 \times 10^{-2} \mathrm{r} / \mathrm{min}$ in a measurement time of only $80 \mathrm{~ms}$. The angular range is limited by the potentiometer geometry and the finite size of the sliding electrode, and typically amounts to about $270^{\circ}$. The sensor system also indicates the moving direction.
\end{abstract}

Index Terms - Low-speed measurement, noncontact resistive potentiometer, potentiometric sensor, relaxation oscillator.

\section{INTRODUCTION}

$\mathbf{S}$ PEED is one of the quantities to be measured in many applications, such as those with robots, rubber mills, and paper mills. Many digital methods for the measurement of speed have been presented [1]-[4]. These methods are very suitable for the measurement of speed within a range of $10-10000 \mathrm{r} / \mathrm{min}$. However, for the measurement of low speed, for instance lower than $1 \mathrm{r} / \mathrm{min}$, these methods require a relatively long measurement time because at least one rising edge of the pulse signal from the speed-sensing element should be detected during a measurement time. A simple alternative and relatively faster method for the measurement of very low speed can be obtained by using a potentiometer for the sensing element [5]. Using this method, an accuracy of $0.8 \%$ has been obtained for a speed of $1 \mathrm{r} / \mathrm{min}$ in a measurement time of $200 \mathrm{~ms}$. However, since the sliding contact is directly in contact with the resistive element of the potentiometer, the long-term stability is bad and there is a hysteresis when the speed (absolute value and moving direction) is changed. These drawbacks can be overcome with the noncontact resistive potentiometer, which was recently described for position sensors [6], [7].

In this paper, we describe the use of the noncontact resistive potentiometer for the measurement of low speed in a short

Manuscript received March 1, 1995; revised December 4, 1998.

The authors are with the Faculty of Information Technology and Systems, Delft University of Technology, Delft, The Netherlands.

Publisher Item Identifier S 0018-9456(98)09881-7.

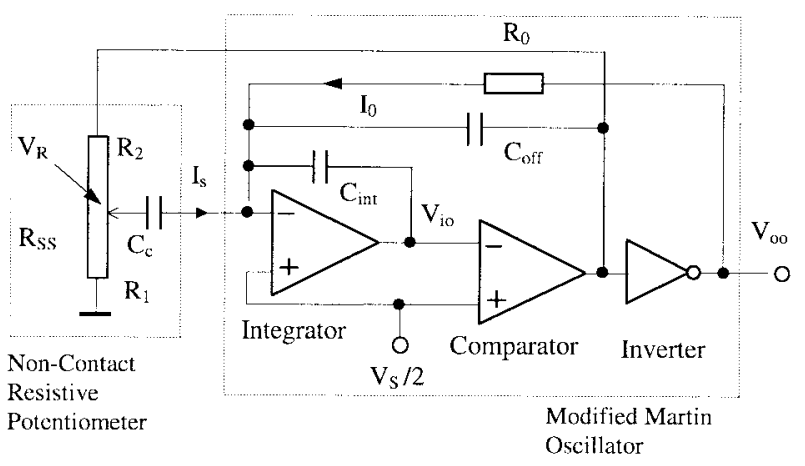

Fig. 1. The principle of the novel noncontact resistive potentiometric sensor.

measurement time. By using a novel algorithm, the problems of measuring nonconstant physical quantities are solved. It is shown that the influences of the many nonidealities of the measurement system are also eliminated or reduced. Meanwhile, the direction of movement can be indicated.

\section{SENSOR PRINCIPLE}

Fig. 1 shows the basic principle of the noncontact potentiometric speed sensor system. It mainly consists of a noncontact resistive potentiometer [6], [7] and an oscillator which is described in [8] and [9], and which is called the modified Martin oscillator, referring to an oscillator designed earlier by Martin [10].

The sensing element for low speed is a modified resistive potentiometer with a sliding contact which is not directly in contact with the resistive layer of the potentiometer [6]. It consists mainly of two parts: a moving electrode and a resistive layer. A coupling capacitor $C_{c}$ is formed between the sliding electrode and the resistive layer. The sum of the resistances $R_{1}$ and $R_{2}$ is the resistance $R_{S S}$ of the resistive layer. Although the sliding electrode has a finite size, with respect to its position, it can be considered as a point contact in the average position. The movement of the sliding electrode results in the change of the current $I_{s}$ through capacitance $C_{c}$.

The current $I_{s}$ which carries the information of the speed to be measured can be converted into period-modulated signals by using a relaxation oscillator, for instance the modified Martin oscillator. A microcontroller can directly decode the period-modulated signals from the output of the oscillator. 


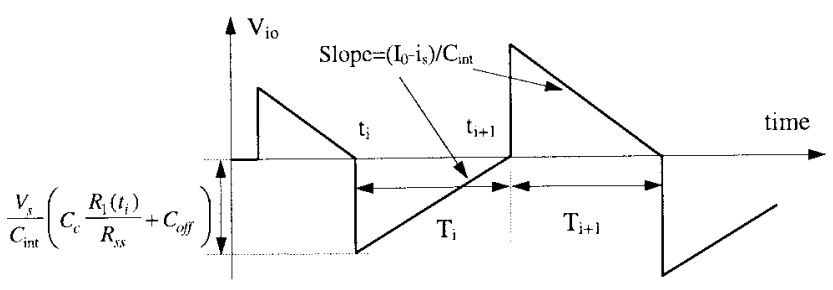

Fig. 2. The $V_{i o}$ waveform of the oscillator.

Fig. 2 shows the waveform of the voltage $V_{i o}$ at the integrator output of the oscillator for the case in which the sliding electrode of the sensing element is moving.

According to the principle of the oscillator [8]-[10], two adjacent charge and discharge time intervals $T_{i}$ and $T_{i+1}$ amount to

$$
\begin{array}{r}
T_{i}=\frac{V_{s}\left(C_{c} \frac{R_{1}\left(t_{1}\right)}{R_{s s}}+C_{\text {off }}\right)}{I_{0}-i_{s}} \\
T_{i+1}=\frac{V_{s}\left(C_{c} \frac{R_{1}\left(t_{i+1}\right)}{R_{s s}}+C_{\text {off }}\right)}{I_{0}-i_{s}}
\end{array}
$$

where $V_{s}$ is the supply voltage and $i_{s}$ is a current which is caused by the change of the resistance with time. For the current $i_{s}$ it holds that

$$
i_{s}=C_{c} \frac{d V_{R}}{d t}=\frac{V_{s} C_{c}}{R_{s s}} \cdot \frac{d R_{1}(t)}{d t} .
$$

In the case of a linear potentiometer, for the instantaneous speed $\vartheta$, it holds that

$$
\vartheta=\frac{L_{e}}{R_{S S}} \cdot \frac{d R_{1}(t)}{d t}
$$

where the coefficient $L_{e}$ is the effective length of the linear resistive layer so that (3) can be rewritten as

$$
i_{s}=\frac{V_{s} C_{c}}{L_{e}} \vartheta
$$

For a constant speed, the relation between resistances $R_{1}\left(t_{i}\right)$ at moment $t_{i}$ and $R_{1}\left(t_{i+1}\right)$ at moment $t_{i+1}$ is

$$
R_{1}\left(t_{i+1}\right)=R_{1}\left(t_{1}\right)+\frac{d R_{1}}{d t} T_{i}
$$

From (1)-(6), for the measured speed $\vartheta$ over time $T_{i}+T_{i+1}$, it is found that

$$
\vartheta=\frac{I_{0} L_{e}}{V_{s} C_{c}} \cdot \frac{T_{i+1}-T_{i}}{T_{i+1}}=\frac{L_{e}}{2 R_{0} C_{c}} \cdot \frac{T_{i+1}-T_{i}}{T_{i+1}} .
$$

By measuring two adjacent half periods with (7), the speed over the time interval $T_{i}+T_{i+1}$ can be obtained. Because the time interval for one successive measurement $\left(T_{i}+T_{i+1}\right)$ can be relatively short, (7) is also used as an approximation for the case of slowly changing speed. It can be shown that this measuring method has some drawbacks due to:

- the error caused by the delay time $t_{d}$ of the processing circuit,

- the serious influence of offset voltage and bias current of the integrator and comparator,
- the large sampling noise, especially in the case of a very short measuring time.

These drawbacks can be overcome by measuring many full periods of the output signal of the oscillator. We are supposing that $T_{11}$ and $T_{22}$ represent two adjacent measurement time intervals for $N$ full periods of the oscillator, respectively, where

$$
\begin{aligned}
& T_{11}=\sum_{i=1}^{2 N} T_{i} \\
& T_{22}=\sum_{i=2 N+1}^{4 N} T_{i} .
\end{aligned}
$$

After some calculations using (1)-(6), (8), and (9), it can be shown that

$$
\frac{T_{11}}{T_{22}}=\left(1-\frac{V_{S} C_{c}}{I_{0} L_{e}} \vartheta_{\mathrm{av}}\right)^{2 N} .
$$

From (10), for the measured average speed $\vartheta_{\text {av }}$ over time $T_{11}+T_{22}$, it is found that

$$
\vartheta_{\mathrm{av}}=\frac{I_{0} L_{e}}{V_{s} C_{c}}\left(1-\sqrt[2 N]{\frac{T_{11}}{T_{22}}}\right)=\frac{L_{e}}{2 R_{0} C_{c}}\left(1-\sqrt[2 N]{\frac{T_{11}}{T_{22}}}\right) .
$$

By measuring the two adjacent time intervals for $N$ full periods $T_{11}$ and $T_{22}$, the speed can be calculated with (11). Meanwhile, the sign of the result of (11) indicates the moving direction.

\section{MEASUREMENT MethoD}

Equation (11) shows that the measured speed is not only related to the measured periods but also to the values of devices $R_{0}$ and $C_{c}$. By using some additional measurements, this influence can be eliminated. Fig. 3 shows a complete electronic circuit of the low-cost noncontact resistive potentiometric sensor for the measurement of low speed. The signal processor is composed of a modified Martin oscillator [8], [9] and a selector formed by two NAND gates. The selector is used in the four possible output states to provide the alternation of the terminals of the sensing element and to enable the offset measurements. The microcontroller controls the selector and acquires the output data from the oscillator. Table I shows the controlled states for the selector in a complete measurement process.

The states 1-0 for $T_{11}$ and $T_{22}$ in the table correspond to the connection of the noncontact resistive potentiometer as shown in Fig. 1. As an alternative, the states $0-1$ for $T_{11}$ and $T_{22}$ also could be used, but this does not make any difference. In the table, $T_{11}, T_{22}, T_{C c}$, and $T_{\text {off }}$ represent, respectively, time intervals for $N$ full periods of the oscillator in the different states of the output signals of the NAND gates, where

$$
\begin{aligned}
& T_{C c}=\frac{2 N V_{s}\left(C_{c}+C_{\mathrm{off}}\right)}{I_{0}}=4 N R_{0}\left(C_{c}+C_{\mathrm{off}}\right) \\
& T_{\mathrm{off}}=\frac{2 N V_{s} C_{\mathrm{off}}}{I_{0}}=4 N R_{0} C_{\mathrm{off}} .
\end{aligned}
$$

These two periodic signals have the same waveform which is shown in Fig. 2, but the values of these two periods do 


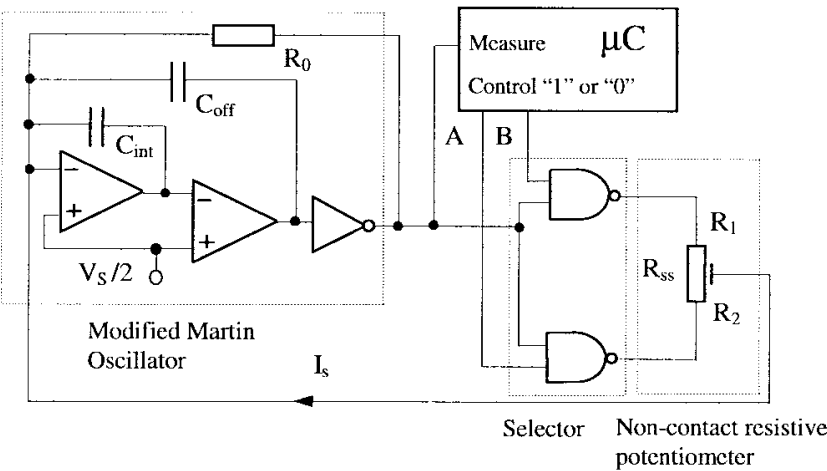

Fig. 3. A complete electronic circuit for the measurement of low speed.

TABLE I

The Controlled States For the Selector in a Complete Measurement Process

\begin{tabular}{c|c|c|c|c}
\hline Time interval to be measured & $T_{I 1}$ & $T_{22}$ & $T_{C}$ & $T_{o f f}$ \\
\hline Corresponding control states A-B & $1-0$ & $1-0$ & $1-1$ & $0-0$ \\
\hline
\end{tabular}

not change with the speed and the position of the moving electrode. The measurement for the time intervals $T_{C c}$ and $T_{\text {off }}$ determines the value of $R_{0} C_{c}$. For the average speed $\vartheta_{\mathrm{av}}$ over the complete measurement time $T_{m}=T_{11}+T_{22}+T_{C c}+T_{\text {off }}$, it can be found that

$$
\vartheta_{\mathrm{av}}=\frac{2 N L_{e}}{T_{C c}-T_{\mathrm{off}}}\left(1-\sqrt[2 N]{\frac{T_{11}}{T_{22}}}\right) .
$$

From (14), it can be calculated that the measured speed $\vartheta_{\mathrm{av}}$ is independent of the capacitance $C_{c}$. This is an important property because it means that the measurement is immune to the electrode distance and the mechanical tolerances which result from deviations or nonuniformity of the electrode distance.

\section{The INFLuENCE OF Systematic ERRors}

\section{A. The Influence of Some Nonidealities of the Signal Processor}

Fig. 4 shows some main nonidealities of the signal processor, where $I_{\text {bias }}$ is the input bias current of the input amplifier (integrator), and $V_{\text {off1 }}$ and $V_{\text {off2 }}$ are respectively the input offset voltages of the amplifier and the comparator. The capacitance $C_{p}$ represents the parasitic capacitance of the input terminal of the integrator and the cable wires. The current source $I_{0}$ is controlled by the output signal of the oscillator. The current $i_{s}$ is caused by the change of the resistance $R_{1}$, as described by (3).

If $I_{0}$ is an ideal current source, the offset voltage $V_{\text {off1 }}$ only causes a dc. level shift of some of the signals without changing the period of the oscillator. If $I_{0}$ is a current source with a finite impedance, the offset voltage $V_{\text {off1 }}$ causes an additional influence which is similar to that of the bias current $I_{\text {bias }}$. The offset voltage $V_{\text {off2 }}$ only causes a dc level shift of some of the signals without changing the period of the oscillator. The influences of the bias current $I_{\text {bias }}$ which includes the contribution of the offset $V_{\text {off } 1}$, and the time delay $t_{d}$ of the integrator and the comparator are now discussed. Fig. 5 shows the output signals of the oscillator.

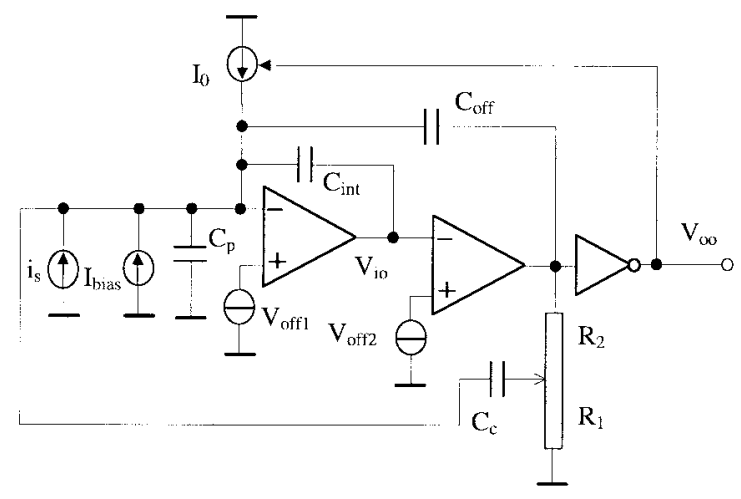

Fig. 4. Some main nonidealities sources in the signal processor.

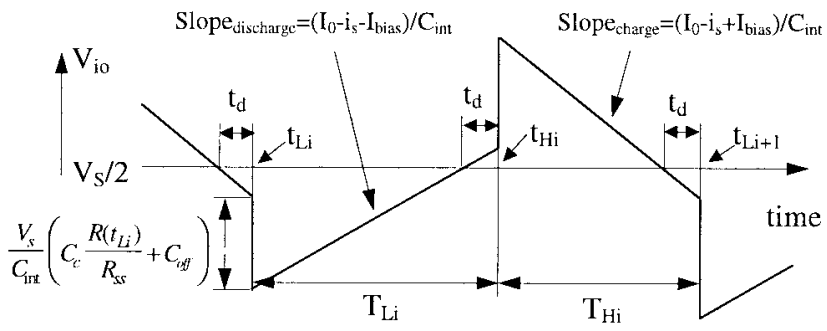

Fig. 5. The output voltage of the integrator.

Extension of (1) and (2) to include the effects of delay time and bias current enables us to find the relative errors $\varepsilon_{\text {rel }}$ which is defined as

$$
\varepsilon_{\mathrm{rel}}=\frac{\vartheta_{\mathrm{av}}-\vartheta_{\mathrm{av} 0}}{\vartheta_{\mathrm{av} 0}}
$$

where $\vartheta_{\text {avo }}$ denotes the value of the average speed in the ideal case. For this relative error, after some straightforward calculations, it is found that

$$
\varepsilon_{\text {rel }} \cong \frac{I_{\text {bias }}^{2}}{2\left(I_{0}^{2}-I_{\text {bias }}^{2}\right)}\left(1-\sqrt[2 N]{\frac{T_{22}}{T_{11}}}\right)
$$

From (16), it can be concluded that the time delay $t_{d}$ of the integrator and the comparator does not affect the measured speed and that the bias current $I_{\text {bias }}$ only causes a secondorder effect. A large value of the current $I_{0}$ helps to reduce the influence of the bias current $I_{\text {bias }}$.

\section{B. The Influence of the Slew Rate of the Op-amp}

Fig. 6 shows the slewing effect, where $R_{0}$ is the impedance of the current source.

Due to the slew rate of the op-amp, the duration of the slewing $T_{s l}$ for the output of the integrator is

$$
T_{s l}=\frac{V_{i o}}{S_{R}}=\frac{C_{\mathrm{off}}}{C_{\mathrm{int}}} \cdot \frac{V_{s}}{S_{R}}
$$

where $S_{R}$ is the slew rate of the op-amp. During slewing, the input voltage at the inverting input will be nonzero which reduces the current $I_{0}$ by a linear amount when the impedance of the current source is finite. This results in the changes of the periods of the oscillator. After some calculations, the relative 


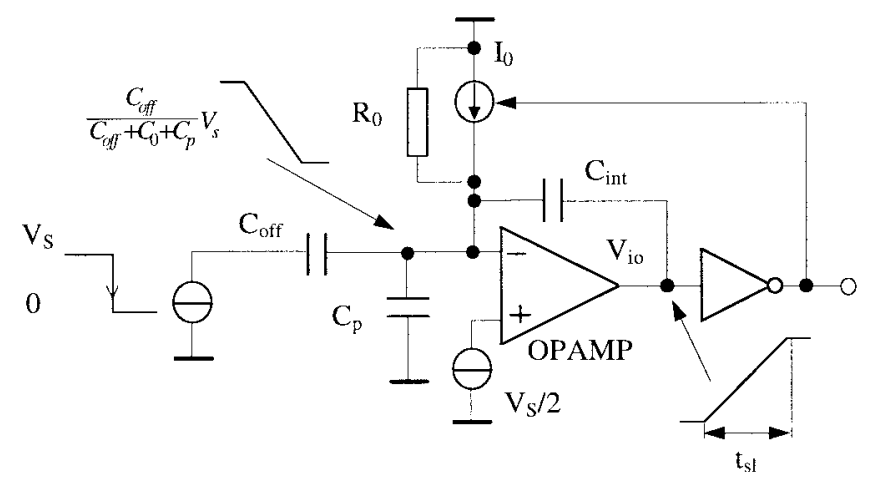

Fig. 6. The effect of the slewing.

error due to the slewing effect of the op-amp is found

$$
\begin{aligned}
\varepsilon_{\mathrm{rel}} \cong & \frac{V_{s}}{2 S_{R} R_{0} C_{\mathrm{int}}\left(C_{\mathrm{off}}+C_{\mathrm{int}}+C_{p}\right)} \\
& \cdot\left[\frac{3 C_{\mathrm{off}}}{2 N}\left(\sqrt[2 N]{\frac{T_{11}}{T_{22}}}\right)+2 C_{\mathrm{off}}+C_{c}\right] .
\end{aligned}
$$

Equation (18) shows that for a small slewing effect, the slew rate $S_{R}$ of the op-amp and the integration capacitance $C_{\text {int }}$ should be as large as possible. A large parasitic capacitance $C_{p}$ also reduces the slewing effect. But this will affect the period of the oscillator. A very large impedance of the current source will also reduce the slewing effect of the op-amp.

\section{The Influence of the Finite Bandwidth of the Op-amp}

The input impedance of the integrator (Fig. 4) amounts to

$$
R_{\mathrm{in}} \cong \frac{1}{\omega_{1} C_{\mathrm{int}}}
$$

where $\omega_{1}$ is the unity-gain frequency of the op-amp. In this case, the input impedance $R_{\text {in }}$ is a resistor with frequency-independent magnitude. Because of this nonzeroinput impedance, the periods of the oscillator are changed. This causes a relative error, which in fact is due to the finite bandwidth of the op-amp, which equals

$$
\varepsilon_{\mathrm{rel}} \cong \frac{I_{0}}{\omega_{l} C_{\mathrm{int}} V_{s}}\left(1-\sqrt[2 N]{\frac{T_{11}}{T_{22}}}\right)
$$

Equation (20) shows that for a small bandwidth effect, the unity-gain frequency $\omega_{1}$ of the op-amp, the integration capacitance $C_{\text {int }}$, and the voltage supply $V_{s}$ should be as large as possible, and the current $I_{0}$ should be as small as possible. But for a small $I_{0}$ other nonidealities, such as the influence of the bias current, will be increased. Therefore, the optimal value of $I_{0}$ is a compromise between the various nonidealities.

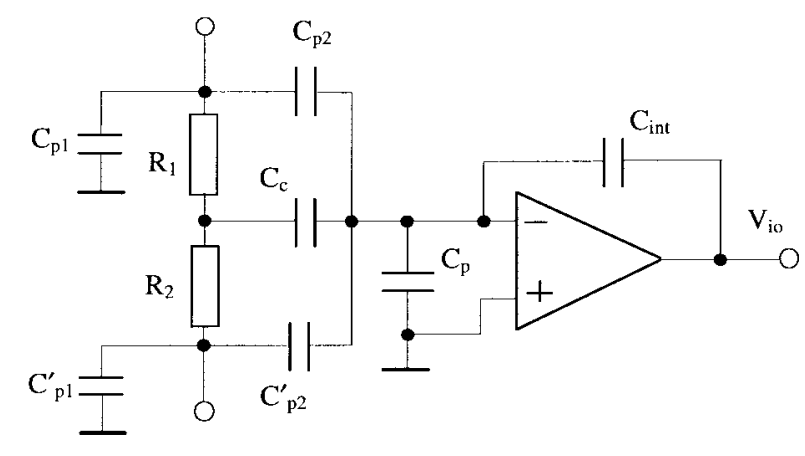

Fig. 7. The parasitic capacitors of the sensor.

\section{The Influence of the Parasitic Capacitance}

The parasitic capacitors of the sensor are shown in Fig. 7.

The effect of the parasitic capacitor $C_{p}$ on the measured speed has been shown in (18). On the other hand, $C_{p}$ affects the periods of the oscillator due to the limited bandwidth of the op-amp, so $C_{p}$ also affects the measured speed by (20). The effects of the parasitic capacitors $C_{p 1}$ and $C_{p 1}^{\prime}$, which include the cable capacitances, can be ignored. The parasitic capacitances $C_{p 2}$ and $C_{p 2}^{\prime}$ are also composed of two parts: the parasitic capacitances between the sliding electrode and the terminals of the resistive layer, and the parasitic capacitances between the outputs of the selector and the inverting input of the integrator in the measuring circuit.

The capacitances $C_{p 2}$ and $C_{p 2}^{\prime}$ cause an influence on the measured speed via the $\left(T_{C c}-T_{\text {off }}\right)$ in (14) only. When this effect is taken into account, then for the measured speed $\vartheta_{\text {av }}$ it is found that

$$
\vartheta_{\mathrm{av}}=\left(1+\frac{C_{p 2}+C_{p 2}^{\prime}}{C_{c}}\right) \frac{2 N L_{e}}{T_{C e}-T_{\mathrm{off}}}\left(1-\sqrt[2 N]{\frac{T_{11}}{T_{22}}}\right)
$$

Equation (21) shows that the measured speed is related to the capacitance $C_{c}$ due to the effect of the parasitic capacitances. Therefore, the parasitic capacitances $C_{p 2}$ and $C_{p 2}^{\prime}$ should be as low as possible.

\section{Limitations of THE MEASUREMENT RANGE}

\section{A. Resolution and Minimum Measurable Speed}

The resolution and minimum measurable speed of the system are mainly determined by the noise of the system. The noise mainly originates from the oscillator and the quantized measurement of the period with a microcontroller (sampling noise). In the cases when the sliding electrode of the sensing element is fixed (speed $\vartheta=0$ ) for the standard deviation $\sigma_{s p}$ of the noise in the speed measurement, it holds that [11] (see (22), shown at the bottom of the page) where $f_{T}$ is the bandwidth of the op-amp, $v_{\text {eq }}$ is the equivalent input noise

$$
\sigma_{s p} \cong \frac{2 L_{e}}{T_{m}-T_{C c}-T_{\mathrm{off}}} \sqrt{\frac{C_{p}}{\left(C_{c}+C_{\mathrm{off}}\right)^{2}} \cdot \frac{2 f_{T} C_{\mathrm{int}} V_{\mathrm{eq}}^{2}}{V_{s}^{2} N}+\frac{2 t_{c}^{2}}{3\left(T_{m}-T_{C c}-T_{\mathrm{off}}\right)^{2}}}
$$




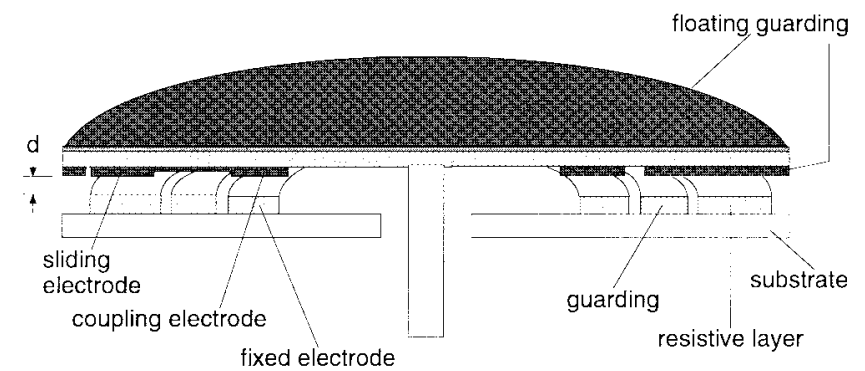

Fig. 8. The physical structure of the noncontact resistive potentiometer.

$\left(\mathrm{V} / \mathrm{Hz}^{1 / 2}\right)$ of the op-amp and $t_{c}$ is the sampling period of the microcontroller.

\section{B. The Maximum Measurable Speed}

Generally, the maximum measurable speed is mainly limited by the frequency $f_{\text {osc }}$ of the oscillator. The time required for one successive measurement is always longer than the $4 / f_{\text {osc }}$, so the maximum measurable speed is

$$
\vartheta_{\max } \leq L_{e} / T_{m} .
$$

Example: For an angular-speed sensor with an angular length of $270^{\circ}(3 \pi / 4)$, when $T_{m}=80 \mathrm{~ms}, T_{m}-T_{C c}-T_{\text {off }}=$ $50 \mathrm{~ms}, C_{c}=2.2 \mathrm{pF}$ and $\sigma_{s p}=0.08 \mathrm{r} / \mathrm{min}$ (experimental value), the measurable range of the sensor amounts to: 0.08 $\mathrm{r} / \mathrm{min} \leq \vartheta_{\mathrm{av}} \leq 562 \mathrm{r} / \mathrm{min}$.

\section{EXPERIMENT RESULTS}

The prototype presented in [6], [7] for the angular-position sensing system has been used for sensing angular speeds. It consists of a noncontact resistive potentiometer, a signalprocessing circuit and a microcontroller of the type INTEL D87C51FB. The signal-processing circuit uses a modified Martin oscillator [6], [8] which has been implemented with a simple dual op-amp (TLC272AC) and two CMOS NAND gates according to the circuit shown in Fig. 3.

The structure of the noncontact resistive potentiometer is shown in Fig. 8. In this sensing element, the potentiometric resistance $R_{S S}$ is about $100 \mathrm{k} \Omega$. The sliding electrode was made using printed circuit-board technology and has an effective area of $61 \mathrm{~mm}^{2}$. The distance between the sliding electrode and the resistive layer is about $0.2 \mathrm{~mm}$ which results in an equivalent capacitance $C_{c}$ of about $2.2 \mathrm{pF}$. To reduce the influence of the parasitic capacitors and of electromagnetic interference, the sliding electrode is surrounded by a guarding electrode, while shielded wires are used.

The measuring system is powered by a single $5 \mathrm{~V}$ supply voltage. The frequency $\left(1 / T_{\text {off }}\right)$ of the oscillator during the offset measurement is about $7.0 \mathrm{kHz}$. During the other measurement phases, the oscillator frequency is in the range of about 3.3-7.0 kHz. For the different measurement times, the measured noise of the angular-speed sensor, including the noise of the signal processor, the sampling noise of the microcontroller, and the noise of the sensing element, is shown in Fig. 9.

The implemented angular-speed sensor was employed to measure two constant rotating speeds of about 9.5 and 33.5

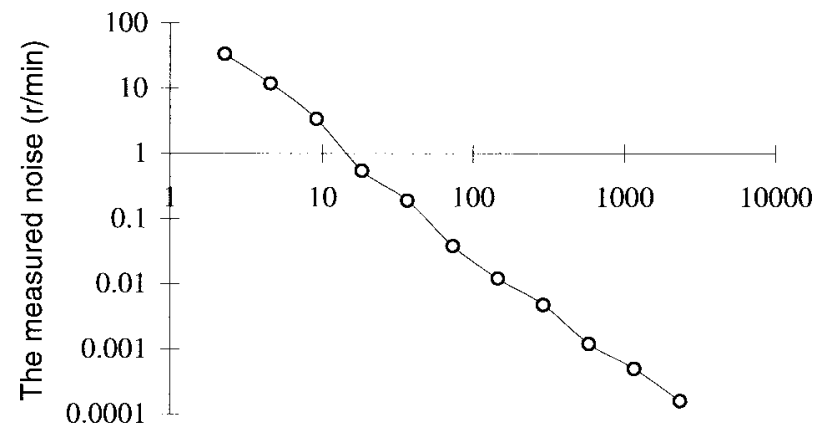

The measurement time (ms)

Fig. 9. The measured noise of the angular-speed sensor at the different measurement times: The results of the measurements performed under identical conditions in a fixed position of the sliding electrode.

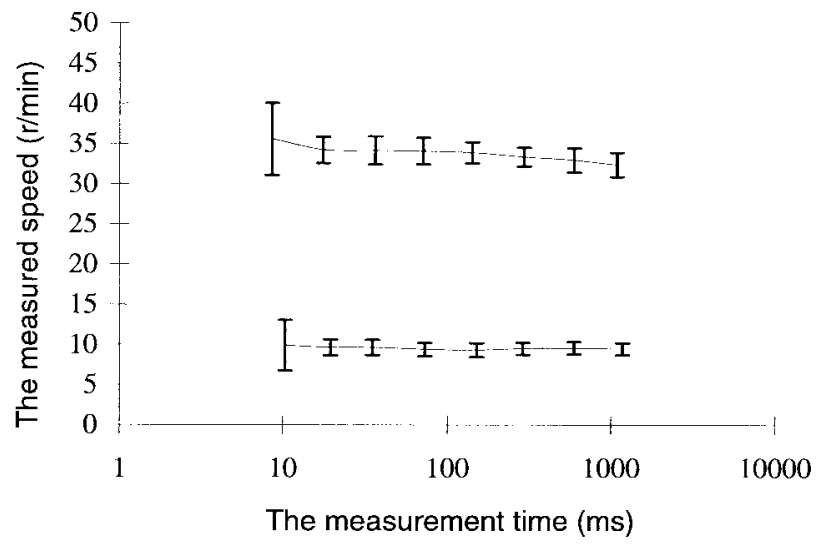

Fig. 10. Measurement results for two constant speeds: showing the measured speeds and errors as function of the measurement times.

$\mathrm{r} / \mathrm{min}$ at the different measurement times. The measurement results are shown in Fig. 10. Meanwhile, the standard deviations of these speed measurements are shown. It is concluded that the standard deviation for a short measurement time is mainly originated from the sampling noise of the microcontroller, the standard deviation for a relatively long measurement time is mainly originated from the mechanical noise of the sensing element and the inaccuracy of the speed source.

\section{CONCLUSIONS}

A novel low-cost noncontact resistive potentiometric sensor for the measurement of low speed has been presented. The system can be realized using low-cost components. The performance of the system is insensitive to the main nonidealities of the electronic components. The mechanical part of the sensing element consists of a circular or linear potentiometer where the sliding electrode is capacitively coupled to the resistive layer. By applying an improved algorithm, the low speed can be measured with a relatively high resolution in a short measurement time. A prototype of the system has been built and tested. The resolution of the system is mainly limited by the sampling noise and the mechanical noise of the sensing element. As compared to existing speed sensors, the main advantages of this novel low-speed sensor are its simplicity, low cost, and the low speed that can be measured with a 
relatively high resolution in a short measurement time. The angular range typically amounts to about $270^{\circ}$ and is limited by the potentiometer geometry and the finite size of the sliding electrode.

\section{REFERENCES}

[1] R. Bonert, "Design of a high performance digital tachometer with a microcontroller," IEEE Trans. Instrum. Meas., vol. 38, pp. 1104-1108, Dec. 1989.

[2] Y. Ameari and I. Masuda, "Velocity sense detection based on the spatial filter method," IEEE Trans. Instrum. Meas., vol. 39, pp. 649-652, Aug. 1990.

[3] C. D. diCenzo, B. Szabados, and N. K. Sinha, "Digital measurement of angular velocity for instrumentation and control," IEEE Trans. Ind. Electron. Contr. Instrum., vol. IECI-23, pp. 83-86, Feb. 1976.

[4] M. Prokin, "DMA transfer method for wide-range speed and frequency measurement," IEEE Trans. Instrum. Meas., vol. 42, pp. 842-846, Aug 1993.

[5] W. Ahmad and M. Ahmad, "A potentiometric transducer for the measurement of very low speeds," IEEE Trans. Instrum. Meas., vol. IM-34, pp. 470-471, Sept. 1985

[6] X. Li and G. C. M. Meijer, "A novel smart resistive-capacitive position sensor," IEEE Trans. Instrum. Meas., vol. 44, pp. 768-770, June 1995.

[7] X. Li, "Low-cost smart capacitive sensors for position and speed measurement," Ph.D. dissertation, Dept. Elect. Eng., Delft Univ. of Technology, Delft, The Netherlands, May, 1997.

[8] F. N. Toth and G. C. M. Meijer, "A low-cost, smart capacitive position sensor," IEEE Trans. Instrum. Meas., vol. 41, pp. 1041-1044, Dec. 1992.

[9] J. van Drecht, "Relaxatie oscillator," Patent 9101 076, The Netherlands, Jan. 1992.

[10] K. Martin, "A voltage-controlled switch-capacitor relaxation oscillator," IEEE J. Solid-State Circuits, vol. SC-16, pp. 412-414, 1981.

[11] F. M. L. van der Goes, "Low-cost smart sensor interfacing," Ph.D. dissertation, Delft Univ. Technology, Delft, The Netherlands, 1996.

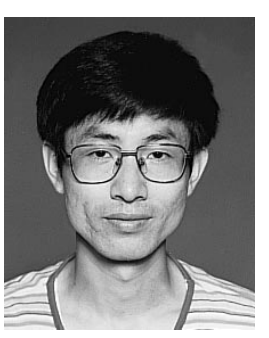

Xiujun Li was born in Tianjin, China, on February 19, 1963. He received the B.Sc. degree in physics and the M.Sc. degree in electrical engineering from Nankai University, Tianjin, China in 1983 and 1986, respectively. In 1997, he received the Ph.D. degree in electrical engineering from Delft University of Technology, Delft, The Netherlands.

In 1996, he was an Assistant Researcher at the Department of Electrical Engineering, Delft University of Technology, where he was involved in research and the development of smart capacitive sensors and low-cost interfaces for smart sensors. Since May 1997, he has worked for SMARTEC B.V. on the smart temperature sensor and smart sensor interface in part time. In 1997, he co-founded the company, G. Wang, where he is a Consultant in the field of sensor systems. His research interests are smart sensors, smart sensor system and smart signal processing.

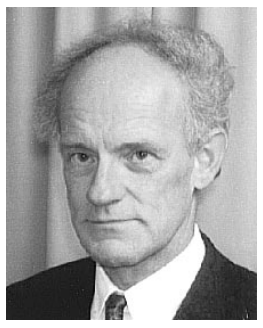

Gerard C. M. Meijer (M'94) was born in Wateringen, The Netherlands, on June 28, 1945. He received the ingenieurs (M.S.) and Ph.D. degrees in electrical engineering from the Delft University of Technology, Delft, The Netherlands, in 1972 and 1982, respectively.

Since 1972, he has been part of the Laboratory of Electronics, Delft University of Technology, where he is an Associate Professor, engaged in research and teaching on analog IC's. In 1984 and during 1985-1987, he was involved in the development of industrial level gauges and temperature transducers. In 1996, he was one of the founders of the company Sensart, where he is a Consultant in the field of sensor systems.

Dr. Meijer is a member of the Netherlands Society for Radio and Electronics. 